\title{
Archéopages
}

Archéopages

Archéologie et société

Hors-série 2 | 2010

Archéologie sans frontières

\section{Ateliers primaires de l'Égypte gréco-romaine : le site de Beni Salama dans le Wadi Natrun}

\section{Marie-Dominique Nenna}

\section{(2) OpenEdition}

1 Journals

Édition électronique

URL : https://journals.openedition.org/archeopages/812

DOI : 10.4000/archeopages.812

ISSN : 2269-9872

\section{Éditeur}

INRAP - Institut national de recherches archéologiques préventives

\section{Édition imprimée}

Date de publication : 1 octobre 2010

Pagination : 122-127

ISSN : $1622-8545$

\section{Référence électronique}

Marie-Dominique Nenna, «Ateliers primaires de l'Égypte gréco-romaine : le site de Beni Salama dans le Wadi Natrun », Archéopages [En ligne], Hors-série 2 | 2010, mis en ligne le 01 octobre 2010, consulté le 23 février 2023. URL : http://journals.openedition.org/archeopages/812 ; DOI : https://doi.org/

$10.4000 /$ archeopages. 812 


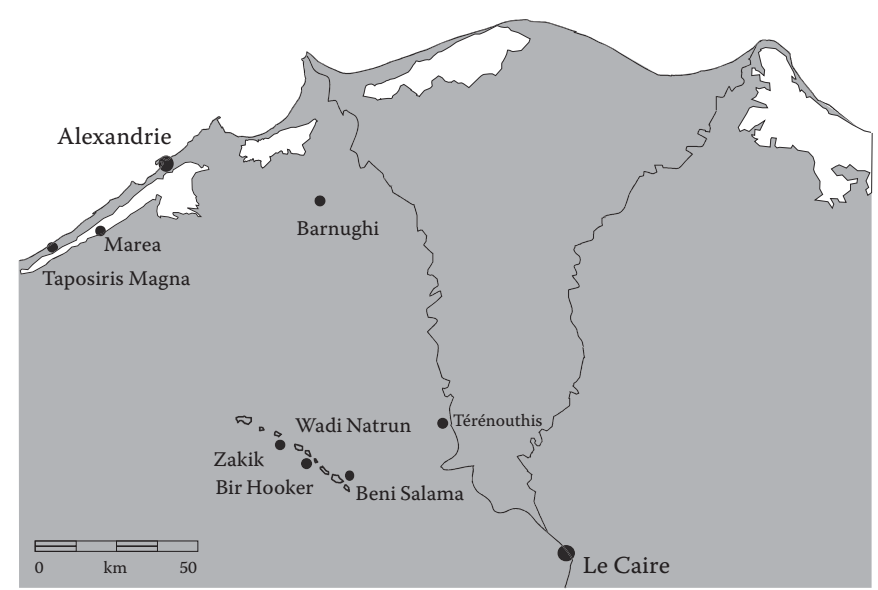

$\approx$

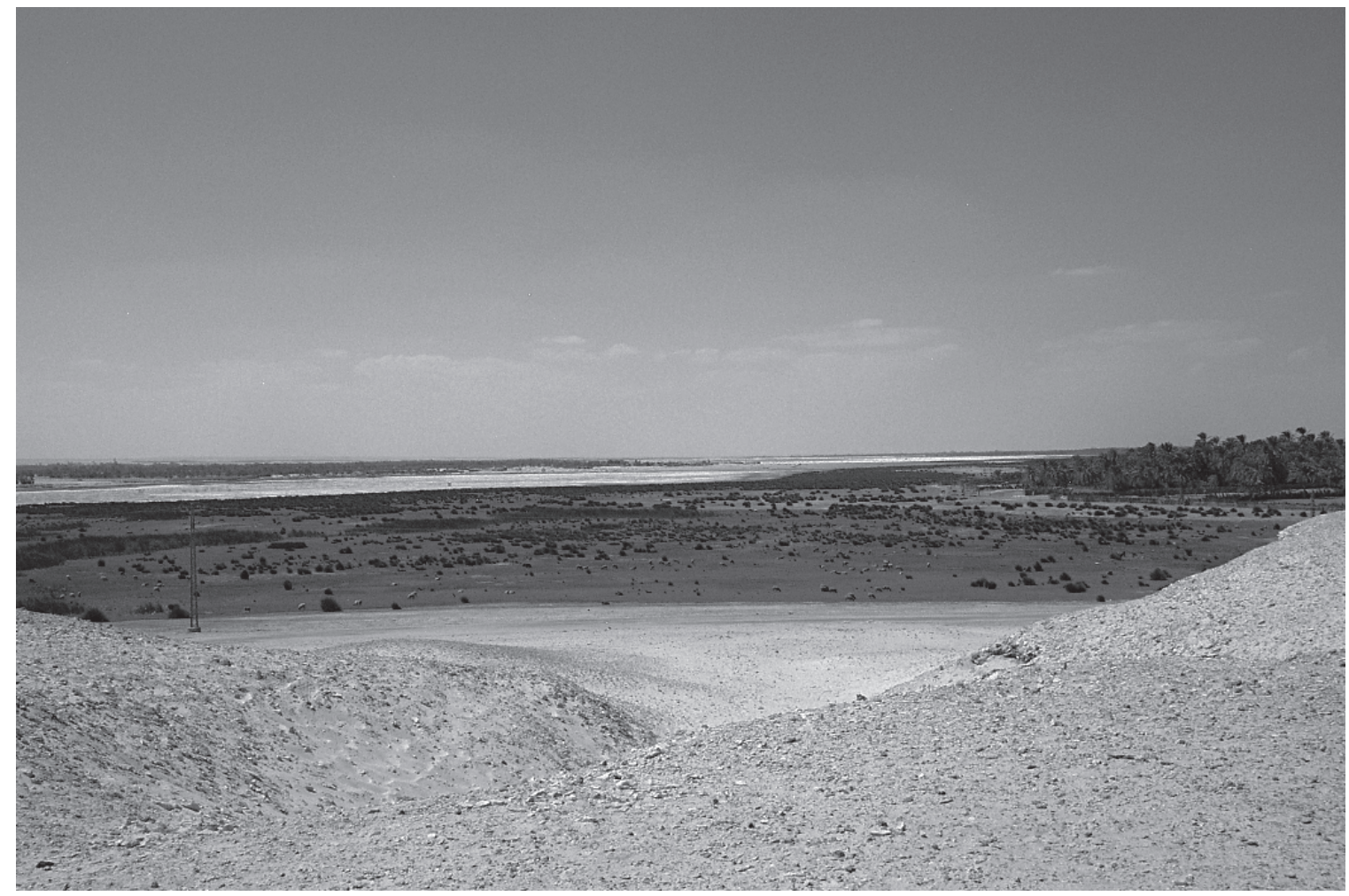

[Fig. 1] Vue du lac Al-Fasidah depuis le plateau dominant le site de Beni Salama. 
003, Mongolie, le premier empire des steppes, Paris, Actes Sud / Mafm, 244. p. ANDRE G., 2002, « Une tombe princière Xiongnu à Gol Mod, Mongolie (campagnes de fouilles 2000-2001) ", Arts Asiatiques, Paris, tome 57, p. 194-205.

Andre G., Holotova-SzineK J., 2009, « Mengu Xiongnu guizu mudi de chubu yanjiu », Kaogu Xuebao, Zhongguo Shehui Kexueyuan, Pékin, p. 69-88.

BATSAÏKHAN Z., 2002, Xiongnu : Archaeology, ethnography and history, National University of Mongolia, Oulan-Bator, édition D. Bayar, 291 p

Brosseder U., 2007, « Zur Archäologie der Xiongnu », in Attila und die Hunnen, catalogue d'exposition du musée de Speyer, Stuttgart, p. 63-71.

Crubézy E., Martin H., Giscard P.-H., Batsaikhan Z., Erdenebaatar S. MAUReille B., Verdier J.-P., 1996, « Pratiques funéraires et sacrifices d'animaux. De la représentativité au symbole. À propos d'une sépulture protohistorique de Mongolie, vallée d’Egiin Gol (région péri-Baïkal) », Paléorient, 22, p. 89-108.

Desroches J.-P. (DIR.), 2001, L'Asie des steppes d'Alexandre le Grand à Gengis khan, catalogue d'exposition, musée national des Arts asiatiques-Guimet, 23 janvier-2 avril, Paris, RMN, $191 \mathrm{p}$

Desroches J.-P., octobre-janvier 2003, «La nécropole de Gol Mod en Mongolie », in Comptes-Rendus de l'Académie des inscriptions et belles-lettres, fasc. III, De Boccard, Paris, p. 1167-1176.

Desroches J.-P., 2005, «Die Welt des Steppe - Das Reich der Xiongnu», in Dchingis Khan und Seine Erben - Das Weltreich der Mongolen, catalogue d'exposition, Bonn, 16 juin 2005- 29 janvier, p. 39-62.

Desroches J.-P., ANDre G. (ÉD.), 2007, Mongolie, les Xiongnu de l'Arkhangaï, Oulan-Bator, Admon, 97 p.

DorJsüren Ts., 2003 (réed.), Arkheologiin Sudalgaa (Mélanges d'études archéologiques), Sh.U.A., Arkheologiin Khureelen, Oulan-Bator, 376 p.

Keyser-Tracoui C., Crubézy E., Ludes B., 2003, «Nuclear and mitochondrial DNA analysis of a 2000-year-old necropolis in the Egyin Gol valley of Mongolia », American Journal of Human Genetics, 73(2), p. 247-60

Miller B., Allard F., Dimaajav E., Lee C., 2006, « A xiongnu tomb complex : excavations at Gol Mod 2 cemetery, Mongolia (20022005) », Mongolian Journal of Anthropology and Ethnology, vol. 2, $\mathrm{n}^{\circ} 2$ (271), p. 1-21.

Prat F., 1980, Les Équidés villafranchiens, CNRS, Paris, Édition du Quaternaire, $154 \mathrm{p}$

RudenKo S. I., 1970, Frozen tombs of Siberia : the Pazyryk burials of Iron-Age horsemen, Londres, J.-M. Dent and sons., 320 p.

Törbat Ts., Amartuvshin Ch., Erdenebat U., 2003, Egiin Golyn sav nutag dakhi arkheologiin dursgaluud, Oulan-Bator, Soyombo, 295 p.

TörвAт Ts., 2004, Khunnugyin jiriin iregdiin bulsh, Oulan-Bator, Soyombo, $110 \mathrm{p}$

TÖRBAT Ts., 2006, «The origin of Xiongnu archaeological culture based on funeral rites ", Mongolian Journal of Anthropology and Ethnology vol. 2, n 2 (271), p. 22-36.

TSEVEendorj D., 1992, Nomads route, International Scientific Expedition - Mongolian Archaeological, Historical and cultural Relics - Mongolia, Oulan-Bator, Ulaanbaatar Press, $430 \mathrm{p}$.

Yerööl-ERdenE CH., 2004, «Gol Modny Khünnü bulshny sudalgaany zarim ür dün», Arkheologiin suddlal, T(II) XXII, fasc. 8, Sh. U. A., Arkheologiin Khureelen, Oulan-Bator, p. 76-109.

Sous presse

Andre G., Holotova-Szinek H., Martin H., " Chevaux et Xiongnu en Mongolie : où donc trouver les cavaliers nomades?", Monumenta Serica, Sankt Augustin, p. 86-119.

Le Gall O., Demars P.-Y., Jarry M., Martin H., Saisons et proies : réflexions sur les notions de prédation(s) et de territoire. Actes du Colloque organisé dans le cadre du XVI ${ }^{\mathrm{e}}$ Congrès de l'UispP, Lisbonne, septembre 2006
Ateliers primaires de l'Égypte gréco-romaine : le site de Beni Salama dans le Wadi Natrun

Marie-Dominique Nenna

CNRS, UMR 5189 " Histoire et Sources des Mondes Antiques"

L a fouille sur les ateliers primaires du Wadi Natrun, qui a débuté en 2003 et s'est close sur le terrain en 2009, a été soutenue par le ministère des Affaires étrangères et européennes, par le laboratoire de rattachement de la directrice de la mission, Marie-Dominique Nenna (UMR 5189, Maison de l'Orient et de la Méditerranée, Lyon) et par le Centre d'études alexandrines (UsR 3134, Alexandrie) ${ }^{\mathbf{1}}$. L'Inrap y collabore par la mise à disposition d'un de ses agents, Aurore Louis, dans le cadre d'un PAS. Cette fouille vient se placer dans un programme plus large, sur lequel Marie-Dominique Nenna travaille depuis une dizaine d'années, qui vise à comprendre quel a été le rôle de l'Égypte dans l'artisanat verrier antique, aussi bien pour la production du verre brut que pour la fabrication des objets finis (vaisselle, contenants, éléments de parure et verre à vitre). Trois types de démarches se trouvent associés dans cette recherche : localisation et examen des sites de production (prospection de surface, géophysique et fouille) ; analyse archéométrique des compositions des verres bruts produits en Égypte; enfin, étude du mobilier mis au jour sur les sites de consommation.

Cadre de l'étude. Depuis une dizaine d'années, on commence à mieux comprendre le système de production et de commerce du verre dans le monde romain. Une division géographique du travail se fait entre les ateliers primaires qui produisent la matière brute à partir de deux composants, le sable et le natron (soude minérale qui permet d'abaisser la température de fusion de la silice de 1700 à $1200^{\circ} \mathrm{C}$, exploitée principalement en Égypte et notamment dans le Wadi Natrun), et les ateliers secondaires qui refondent le verre brut pour en faire des objets. Si le premier type d'atelier est attesté uniquement au Proche-Orient et en Égypte (aussi bien par les découvertes archéologiques que par les recherches archéométriques), le second est commun à l'ensemble de l'Empire romain. Il faut donc supposer un transport de verre brut (depuis les centres orientaux vers les ateliers secondaires de l'ensemble de l'Empire) qui est mis en évidence par une série de cargaisons, dont l'exemple le plus manifeste est l'épave OuestEmbiez, avec son chargement de verre brut, d'objets finis et de verre à vitre, fouillée durant la dernière décennie ${ }^{2}$.

L'intérêt pour les fours primaires des verriers antiques est récent et leur étude en est encore à ses débuts. La découverte en 1992, à Bet Eli'ezer au sud-est de Césarée, d'une série de 17 fours à bassin en a été, en grande partie, à l'origine. C'était en effet la première fois que l'on mettait au jour, et que l'on identifiait comme tels, des fours primaires.
1 Léquipe est constitué Louis (Inrap, 2004-2010), V. Merle (Inrap, 2003 (doctorante, Universite Lyon 2, 2006), V. Pichot (CNRs, Cealex, 2003 doctorante, Université Lyon 2, 2008), E. Thivet de Besançon, 2007), A. Viellet, (Service d'archéologie de Besançon, 2009) d'une architecte (J. Badr, 2007-2010), de deux céramologues, S. Marchand (Ifao, 2003-2010) et S. Marquié et d'une dessinatrice et spécialiste de vannerie (2007-2009). Les levés topographiques ont Shaalan (CNRS, Cealex) et la couverture vidéo

2 Fontaine S.-D. et Foy D., 2007, « L'épave Ouest-Embiez 1, Var : du verre brut et manufacturé en Méditerranée occidentale dans l'Antiquité », Revue archéologique de Narbonnaise, 40, p. 235-268.
GOo, "The Ancient

Glass Industry in Israel: Summary of the Finds in NenNa M.-D. (ÉD.) La Route du verre : ateliers primaires et secondaires millénaire av second au Moyen Âge, Lyon, Travaux de la Maison de l'Orient, 33, p. 49-63 FREESTONE I.C., Gorin-Rosen Y., Hughes M., «Primary Glass from Israel and the Production of Glass in Late Antiquity and the Early Islamic Period», ibidem, p. 71-72 

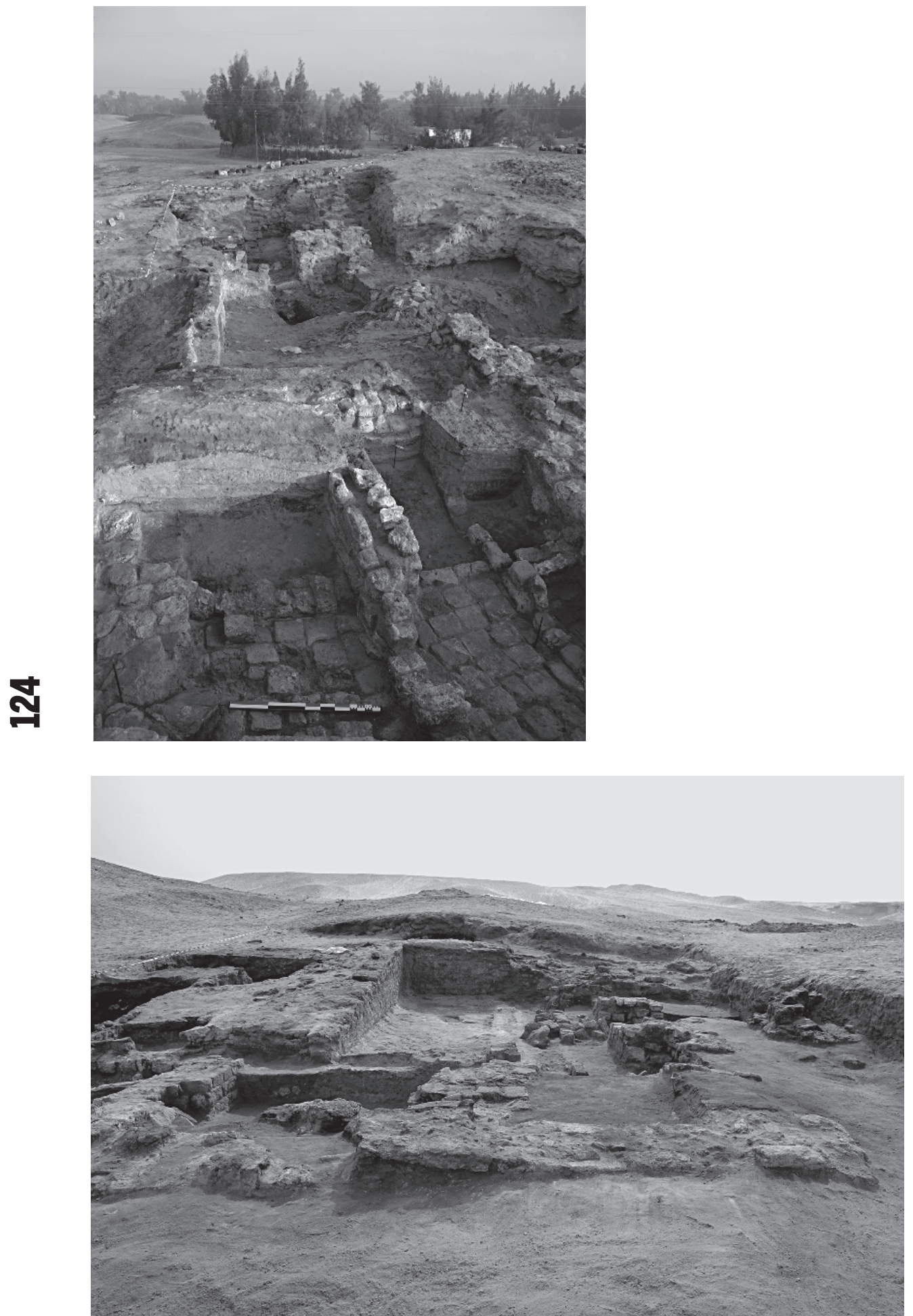

[Fig.2] Vue du secteur 1 depuis le sud avec les deux fours disposés parallèlement. Au fond,

l'emplacement de la chambre de chauffe du four occidental, au deuxième plan, les bassins, au premier plan à gauche, la zone «propre » au sud du bassin.
[Fig.3] Vue du four occidental du secteur 2 depuis le nord. Au premier plan, l'emplacement de la chambre de chauffe, au deuxième plan le bassin, à droite, le dispositif de rampe contre le fantôme du mur démantelé et la zone propre non encore fouillée. 
Ceux-ci sont divisés en deux parties : à l'avant, deux chambres de chauffe de forme triangulaire; à l'arrière, la chambre de fusion, qui mesure $2 \times 4 \mathrm{~m}$, mais seuls les sols des fours sont conservés. Le site de Bet Eli'ezer semble correspondre à une seule phase d'occupation datée des $\mathrm{VI}^{\mathrm{e}}-\mathrm{VII}^{\mathrm{e}}$ siècles. Les verriers ont installé leurs fours, tiré profit des combustibles que la région offrait, puis se sont déplacés vers une autre localité.

Cette fouille a provoqué le réexamen d'une découverte plus ancienne effectuée à Bet She'arim, au sud-est d'Haïfa. À l'intérieur d'une citerne, on avait mis au jour en 1956 une grande dalle de verre de 3,80 x 1,95 m, épaisse de $45 \mathrm{~cm}$. Cette dalle pèse entre 8 et 9 tonnes de verre. L'ensemble avait été daté entre le $\mathrm{IV}^{\mathrm{e}}$ et le VI $\mathrm{I}^{\mathrm{e}}$ siècle. Depuis, une nouvelle étude a proposé, pour des raisons archéologiques et technologiques, de dater cette dalle plus tardivement, du début de l'époque islamique, au $\mathrm{IX}^{\mathrm{e}}$ siècle ${ }^{4}$. Cette dalle est restée en place, car elle est une sorte d'énorme raté. D'autres fours primaires ont été aussi mis au jour dans les années 1950 ainsi que très récemment à Apollonia, toujours datés de la même époque ${ }^{5}$.

Dans tous les cas, ces découvertes se rapportent à la fin de l'Antiquité, si ce n'est au début de l'époque islamique. Seuls les sols des fours sont conservés et leur publication n'offre pas d'éléments archéologiques relatifs à leur élévation et à leur couverture. Ce modèle de four à bassin de fusion perdure, comme le montrent les fours primaires de Tyr, découverts anciennement, mais qui viennent d'être réétudiés et datés des $\mathrm{X}^{\mathrm{e}}$ - $\mathrm{XI}^{\mathrm{e}}$ siècles ${ }^{\mathbf{6}}$, ainsi que l'atelier complet, primaire et secondaire, de Somelaria, situé au nord d'Akko, daté des $\mathrm{XII}^{\mathrm{e}}$-XIII ${ }^{\mathrm{e}}$ siècles?

Ateliers primaires du Wadi Natrun. Face à ces découvertes proche-orientales, il a semblé intéressant, au vu de la réputation des arts verriers en Égypte, d'essayer de repérer de telles structures dans le pays. Les prospections de surface dans la région d'Alexandrie et dans le Wadi Natrun (dépression d'une cinquantaine de kilomètres de long, comprenant des lacs, et qui constitue une sorte d'oasis dans le désert libyque) ont conduit à l'identification de cinq sites d'ateliers primaires, deux en Maréotide (à Taposiris Magna et à Maréa-Philoxénité) et trois dans le Wadi Natrun (à Beni Salama, Bir Hooker et Zakik). À la suite

\footnotetext{
4 BRILl R.H., 1967, « A Great Glass Slab from Ancient Galilee», Archaeology, 20 , p. 88-95; FREESTONE I.C., Gorin-Rosen Y., 1999, « The Great Glass Slab at Bet She'arim, Israel: an Early Islamic Glassmaking Experiment», Journal of Glass Studies, 41, p. 105-116. 5 TAL O., JaCKSON-TaL E. et Freestone I.C., 2004, « New Evidence of the Production of Raw Glass at Late Byzantine Apollonia-Arsuf, Israel », Journal of Glass Studies, 46, p. 51-66.
}

$$
\begin{aligned}
& 6 \text { AlDSWORTH F. et al., } \\
& \text { 2002, « Medieval } \\
& \text { Glassmaking at Tyre, } \\
& \text { Lebanon », Journal of } \\
& \text { Glass Studies, 44, p. 49-66. } \\
& 7 \text { DAVIDSON WEINBERG } \\
& \text { GL., 1987, "A Glass } \\
& \text { Factory of Crusader } \\
& \text { Times in Northern } \\
& \text { Israel», Annales du } \\
& \text { 10 congrès de l'Association } \\
& \text { internationale pour } \\
& \text { l'histoire du verre, } \\
& \text { Madrid-Ségovie, 1985, } \\
& \text { Amsterdam, p. } 305-316 . \\
& \text { 8 Coordonnées } \\
& \text { géographiques (Système } \\
& \text { WGS84) par GPS: } \\
& \text { latitude } 30^{\circ}, 19 m n, 30 s ; \\
& \text { longitude } 30^{\circ}, 25 \mathrm{mn}, 18 \mathrm{~s} \text {. }
\end{aligned}
$$

de prospections géophysiques, une fouille a été ouverte à Beni Salama en $2003^{8}$. Situé au sud du village du même nom, à l'entrée orientale du Wadi Natrun, et au bord du lac Al-Fasidah, ce site se divise en deux parties, au nord deux collines anthropiques, créées par les rejets de l'activité verrière, au sud, sur un plateau, une zone habitée marquée par les vestiges d'un temple du Moyen Empire et une occupation s'étendant depuis la XII ${ }^{\mathrm{e}}$ dynastie jusqu'au viI ${ }^{\mathrm{e}}$ siècle après J.-C.

Guidés par les prospections géophysiques effectuées en 2000 et 2002, nous avons implanté sur la colline sud de la zone artisanale de Beni Salama deux secteurs de fouille, situés à $20 \mathrm{~m}$ de distance, et mis au jour quatre fours à bassin de fusion de verre, de très grande taille, particulièrement bien conservés et tous bien datés, par le mobilier associé, des deux premiers siècles de notre ère. Il s'agit de fours à réverbère (chauffage horizontal) aux murs très épais et construits selon la même orientation vers les vents dominants (nord-ouest). Une dalle de verre pesant entre 15 et 20 t y était produite, à partir d'un mélange de sable et de natron porté à une température de $1200^{\circ} \mathrm{C}$. Une fois la chauffe achevée, l'un des murs longs et une partie de la voûte étaient démantelés, pour pouvoir accéder à la dalle de verre et la débiter en fragments transportables vers les ateliers secondaires. Dans deux cas, le même emplacement a été utilisé par deux fois pour la construction d'un nouveau four, avec une surélévation du sol et des murs existants et une reconstruction de la chambre de chauffe, du mur démantelé et de la couverture.

Dans le secteur $1\left(416 \mathrm{~m}^{2}\right)$, à l'anomalie magnétique en forme de grand rectangle, se situent deux fours disposés parallèlement [Fig.2], les murs opposés des bassins de fusion ayant été démantelés pour accéder à la dalle de verre ; dans le secteur $2\left(260 \mathrm{~m}^{2}\right)$, l'anomalie magnétique en forme d'agrafe correspond à un seul four avec un démantèlement du mur occidental du bassin [Fig.3]. Les configurations des couches de surface des deux secteurs sont différentes. Les fours du secteur 1 sont situés dans un creux de la colline, tandis que ceux du secteur 2 sont implantés sur la pente de la colline ; le secteur 1 a ainsi été transformé en une sorte de bassin, dans lequel les couches supérieures salinisées se sont pétrifiées sur une hauteur allant jusqu'à 1,20 $\mathrm{m}$; dans le secteur 2, une forte érosion due aux vents et aux variations de l'hygrométrie entre la nuit et le jour a entraîné un arasement des structures de plus en plus prononcé du sud vers le nord ; l'épaisseur de la couche salinisée et pétrifiée est beaucoup moins importante. L'histoire des deux secteurs est différente, si le secteur 2 a été abandonné et transformé en dépotoir après la dernière activité verrière, le secteur 1 a été réoccupé en tirant sans doute partie de l'abri que constituait la voûte du dernier four, qui n'avait pas été entièrement démantelée.

Les fours sont constitués de trois éléments principaux : la chambre de chauffe, le bassin de fusion et une zone «propre », dallée le plus 
souvent de briques. Les bassins sont de très grande taille. Dans le secteur 2, les dimensions internes $\mathrm{du}$ four occidental sont de 7,5 $\times 2 \mathrm{~m}$. On ne dispose pas des dimensions du four oriental qui avait été en grande partie détruit; dans le secteur 1, le four occidental présente des dimensions internes de $6,10 \times 2 \mathrm{~m}$, tandis que le four oriental, non entièrement fouillé, est large de $2 \mathrm{~m}$. Les sols, d'une épaisseur d'environ 30 à $40 \mathrm{~cm}$, sont constitués de couches de terre battue, sans doute surmontée d'une assise de briques carrées, et présentent une légère pente ascendante vers le sud. Les murs, construits en briques crues et en briques remployées sont très épais - murs longs de 1,80 à $1,90 \mathrm{~m}$, mur arrière entre $2 \mathrm{~m}$ et 2,50 $\mathrm{m}-$, le mur en contact avec la chambre de chauffe présente une épaisseur de o,40 à o,50 m. L'ensemble était recouvert par une voûte en berceau aplati, constituée de deux types de briques courbes et d'un chemisage, qui présente une pente légèrement descendante vers le sud.

Le bassin de fusion était enterré probablement jusqu'au départ de la voûte sur deux de ses côtés ; sur le troisième côté était aménagée une zone "propre», sans doute réservée au stockage du mélange de sable et natron qui était progressivement versé dans le bassin. L'emplacement de cette zone n'est pas fixe : si, pour le four occidental du secteur 1, elle était toujours placée au sud durant les trois phases d'activité verrière, en revanche, dans le cas du four occidental du secteur 2, elle a été déplacée de l'est vers l'ouest. Des murs de soutènement étaient implantés autour de cette zone propre pour bloquer les remblaiements qui avaient été effectués sur les autres côtés.

La chambre de chauffe est difficile à restituer, vu les maigres vestiges dont on dispose, en raison de l'arasement des structures dans le secteur 2, dues à l'érosion, et, dans le secteur 1 , des reconstructions successives et des perturbations liées à la réoccupation de la zone. Elle semble avoir eu une forme absidale et avoir été divisée en plusieurs compartiments, deux parallèles au côté court du bassin, un ou deux perpendiculaires de chaque côté, avec au moins un compartiment servant de cendrier. On suppose qu'elle était ouverte sur toute sa largeur $(2 \mathrm{~m}$ ) vers le bassin.

L'emplacement du dispositif de tirage reste hypothétique. Aucun vestige n'atteste la construction d'une cheminée sur le mur arrière du bassin, comme on aurait pu s'y attendre. En se fondant notamment sur les exemples des fours primaires de l'Inde d'aujourd'hui, on suppose que la chaleur s'accumulait dans le four et que des orifices qui servaient à la fois au tirage et au versement des matières premières étaient aménagés dans la voûte. On accédait à ces orifices par des rampes partant aussi bien des zones « propres » que de l'espace voisin d'un des longs côtés du bassin.

Reconstitution de la chaîne opératoire. Sable et soude étaient disponibles à proximité. Le plateau, à $50 \mathrm{~m}$ au sud des deux collines, s'implante sur une dune fossile, dont le sable est approprié à la fabrication du verre, même s'il est très pauvre en calcium. Le natron était exploité dans les lacs du Wadi Natrun, entre mi-novembre et mi-mars si l'on se réfère au témoignage de la Description de l'Égypte. On a pu calculer que la quantité de matière première nécessaire devait varier, selon la taille du four, entre 22 et 30 t. Cette masse ne pouvait être chauffée en une seule fois et le mélange de sable et de soude devait être versé tout au long de la cuisson.

La durée de la cuisson et du refroidissement est difficile à restituer et l'on doit se référer aux rares exemples contemporains pour en avoir une idée. Dans le cas de petits fours du royaume de Nupe au Nigéria, elle peut être estimée à environ un jour et demi ; dans celui des fours de beaucoup plus grande taille à Jalesar, entre Agra et Delhi, en Inde, elle s'élève à 30 jours et la matière première est versée dans le bassin tous les trois jours. C'est plutôt avec cette dernière durée qu'il faut comparer le fonctionnement des fours du Wadi Natrun. Cela implique une masse de combustible extrêmement importante pour pouvoir maintenir la chaleur adéquate de $1200^{\circ} \mathrm{C}$ pour la fusion du sable et du natron en verre. Outre le tamaris, l'acacia et le palmier, disponibles dans cette contrée désertique, les verriers recourraient sans doute aux roseaux, combustible à haut potentiel calorifique et disponible en abondance sur les bords des lacs, couramment utilisé dans l'Égypte gréco-romaine, notamment pour le chauffage des bains.

Si l'on additionne les temps nécessaires à la confection des briques de forme variée selon leur emplacement dans le four, à la construction du four lui-même, à la récolte et à la préparation de la matière première et du combustible, à la chauffe et au refroidissement et enfin à la récupération de la dalle de verre et son débitage en fragments transportables, l'ensemble de la chaîne opératoire devait prendre plusieurs mois. En outre, on peut supposer, aussi bien pour des raisons climatiques qu'en raison de l'absence d'habitats structurés dans la zone avoisinante, que la manufacture du verre était saisonnière.

Le verre, une fois réduit en fragments transportables, devait être chargé sur des dromadaires et des ânes et convoyé vers la cité de Térénouthis, située sur la branche occidentale du Nil, où se trouvait le contrôleur du monopole du natron. De-là, il pouvait être emporté par voie fluviale vers Alexandrie et le reste de l'Égypte. Trois types de verres ont été produits dans les ateliers du Wadi Natrun, un verre bleu-vert, un verre décoloré au manganèse et un verre décoloré à l'antimoine. Les analyses chimiques effectuées ces quinze dernières années sur différents sites de l'Empire romain ont montré que ces verres bruts n'avaient pas fait l'objet d'exportation outremer et qu'ils étaient essentiellement destinés au marché égyptien.

Les dimensions des fours mis au jour dans le Wadi Natrun ne cessent d'étonner. Elles impliquent une maitrise technique qui n'avait pas été identifiée jusqu'alors pour une époque aussi ancienne. Les fours à réverbère sont en effet connus dans l'Antiquité, mais ils sont toujours de petite taille. 
La capacité de production des ateliers du Wadi Natrun semble énorme, par rapport à ce qui est attesté jusque-là : en effet, dans un four comme celui du secteur 2 , on produisait une dalle qui devait avoisiner les 15 à $20 t$ de verre brut. Cette avancée technologique se déroulait à la même époque qu'une autre révolution : l'invention du verre soufflé et sa diffusion dans toutes les provinces de l'Empire, qui a fait passer les verres de produits de luxe à objets d'usage quotidien. Une fois la publication achevée dans les Travaux de la Maison de l'Orient, deux voies d'exploration s'ouvriront : prospection à la recherche d'ateliers primaires de verriers dans le Sinaï, dont on sait qu'ils ont fonctionné à partir de la fin du $\mathrm{IV}^{\mathrm{e}}$ siècle et dont le verre brut a alimenté l'ensemble des ateliers de l'Empire romain ; prospection dans les environs d'Alexandrie à la recherche d'officines secondaires, dont les types sont très mal connus en Égypte.

Collet R., Nenna M.-D., 2009, «L'oasis du verre », Les Métiers de l'archéologie, vol. 11, film 22 mn, Alexandrie (en français et en anglais).

Nenna M.-D., Sous presse, « Primary Glass workshops in GraecoRoman Egypt: Preliminary Report on the Excavations of the Site of Beni Salama, Wadi Natrun (2003, 2005-2009) ", in Freestone I, BAILEY J., JACKSON C.M. (ÉD.), Glass in the Roman Empire, in honour of Jennifer Price, Oxford, Oxbow books.

Nenna M.-D., 2007, « Production et commerce du verre à l'époque impériale: nouvelles découvertes et problématiques », FACTA. A Journal of Roman Material Culture, 1, p. 159-181.

NENNA M.-D., 2008, « Nouveaux acquis sur la production et le commerce du verre antique entre Orient et Occident », in Amrein H., Deschler-Erb E., Deschler-Erb S. (Éd.), Congrès International Crafts 2007: Artisanat et Société dans les provinces romaines (Zurich, 2007), Zeitschrift für Schweizerische Archäologie und Kunstgeschichte, 65.1-2, p. 61-65.

Nenna M.-D., Picon M., ViChy M., 200o, « Ateliers primaires et secondaires en Égypte à l'époque gréco-romaine », in NENNA M.-D. (ÉD.), La Route du verre: Ateliers primaires et secondaires du second millénaire av. J.-C. au Moyen Âge, Lyon, Travaux de la Maison de l'Orient, ${ }^{\circ} 33$, p. 97-112.

Nenna M.-D., Picon M., Thirion-Merle V., Vichy M., 2005, "Ateliers primaires du Wadi Natrun: nouvelles découvertes ", Annales du $16^{e}$ congrès de l'Association Internationale pour l'Histoire $d u$ Verre (Londres, 2003), Nottingham, p. 59-63.

\section{La fouille des ateliers du secteur 2}

$$
\begin{aligned}
& \text { Aurore Louis } \\
& \text { Inrap }
\end{aligned}
$$

Ma participation en tant qu'archéologue de l'Inrap à cette mission scientifique dans le Wadi Natrun pour la fouille d'un atelier de verre du Haut Empire repose sur deux points : d'un côté, mes connaissances sur la nature du site fouillé (techniques de fabrication de la matière brute et méthodes de façonnage, typologies, modes et voies de diffusion, habitudes de consommation) et, de l'autre, mes compétences en archéométrie (compositions chimiques du matériau). Ces acquis permettent de mieux appréhender les produits employés, les processus de transformation des composants et la constitution des unités de production.

La fouille des ateliers de verriers s'est avérée complexe, parfois difficile, tant techniquement que scientifiquement. Les conditions climatiques liées au milieu désertique (fortes chaleurs, tempêtes de sable) ont régulièrement ralenti les travaux. Les variations de température et d'humidité ont également eu un impact sur la conservation des structures - notamment les murs en briques crues qui disparaissent très rapidementce qui oblige à fouiller de façon très rapide.

L'enjeu était d'autant plus grand que des ateliers primaires romains étaient mis au jour en Égypte pour la première fois. Aucune comparaison, aucun parallèle avec d'autres structures similaires ne peut être fait. Le travail de documentation sur les fours primaires plus tardifs découverts en Israël et sur les ateliers secondaires d'Europe a permis de se faire une idée globale de l'organisation de ces structures artisanales. Cependant, les ateliers égyptiens se sont révélés relativement différents et la fouille n'a pu être conduite selon un schéma connu et préétabli. Les orientations de fouille ont dû être décidées au fur et à mesure de la découverte des structures. Ainsi, le four du secteur 2 a été appréhendé en cinq temps [Fig.4].

Le travail s'est appuyé sur les données connues par archéo-magnétisme. En 2005, la structure en agrafe (ayant répondu positivement aux tests) ainsi que les structures alentours ont été dégagées et relevées en plan. Ce travail préliminaire a permis d'élaborer un premier plan d'ensemble du secteur, de distinguer plus nettement les contours du four et de discerner le mode d'organisation des structures annexes. Des tests ont ensuite été réalisés au cœur du four, révélant le bassin de fusion, ses parois vitrifiées et son sol marqué des traces laissées par les outils lors de l'extraction de la dalle de verre. Ces sondages ont également permis de déterminer les différentes séquences d'abandon de la structure et surtout de noter la récupération complète du mur occidental du bassin de fusion. Cette découverte s'est avérée importante : elle a permis de comprendre qu'à la fin de la fusion l'un des longs côtés du four devait être enlevé pour faciliter l'accès à la dalle. La suite de la fouille s'est orientée vers les techniques de construction de ce bassin régulièrement démantelé et reconstruit et, surtout, vers la recherche de structures sṕécifiques à cette construction et placées sur la face orientale du bassin.

En 2006, le dégagement en plan du secteur a été poursuivi ; il a révélé la continuation de l'atelier vers le nord et vers l'ouest. Il a permis de mieux cerner le plan du bassin de fusion et de révéler la présence d'une extension riche en cendres correspondant à la chambre de chauffe. Au sud, un sondage réalisé au droit du mur du bassin a montré que ce dernier était ceinturé par un mur de briques crues, régulièrement rehaussé (au gré des différentes cuissons) et que cette partie du four était enterrée.

Au cours de l'année 2007, le dégagement du bassin de fusion et de la chambre de chauffe a été approfondi, tandis que l'aire de fouille a été élargie sur les flancs est et ouest du four. Ces agrandissements ont permis de découvrir les structures bordant le mur ouest du bassin de fusion et de mettre au jour un second bassin de fusion accolé à la face est du premier. Les relations stratigraphiques entre ces deux bassins ont 